University of New Hampshire

University of New Hampshire Scholars' Repository

Space Science Center

Institute for the Study of Earth, Oceans, and

Space (EOS)

9-16-2005

\title{
Further studies of single-sided charge-sharing CZT strip detectors
}

\author{
B Donmez \\ University of New Hampshire - Main Campus \\ John R. Macri \\ University of New Hampshire - Main Campus, John.Macri@unh.edu \\ Mark L. McConnell \\ University of New Hampshire - Main Campus, mark.mcconnell@unh.edu \\ James M. Ryan \\ University of New Hampshire, James.Ryan@unh.edu \\ Mark Widholm \\ University of New Hampshire - Main Campus, Mark.Widholm@unh.edu
}

See next page for additional authors

Follow this and additional works at: https://scholars.unh.edu/ssc

Part of the Astrophysics and Astronomy Commons

\section{Recommended Citation}

Burcin Donmez ; John R. Macri ; Mark L. McConnell ; James M. Ryan ; Mark Widholm ; Tomohiko Narita and Louis-Andre Hamel "Further studies of single-sided charge-sharing CZT strip detectors", Proc. SPIE 5922, Hard X-Ray and Gamma-Ray Detector Physics VII, 59220H (September 16, 2005); doi:10.1117/ 12.617297; http://dx.doi.org/10.1117/12.617297

This Conference Proceeding is brought to you for free and open access by the Institute for the Study of Earth, Oceans, and Space (EOS) at University of New Hampshire Scholars' Repository. It has been accepted for inclusion in Space Science Center by an authorized administrator of University of New Hampshire Scholars' Repository. For more information, please contact Scholarly.Communication@unh.edu. 


\section{Authors}

B Donmez, John R. Macri, Mark L. McConnell, James M. Ryan, Mark Widholm, T Narita, and L A. Hamel 


\title{
Further Studies of Single-Sided Charge-Sharing CZT Strip Detectors
}

\author{
Burçin Dönmez ${ }^{a}$, John R. Macri ${ }^{a}$, Mark L. McConnell ${ }^{a}$, James M. Ryan ${ }^{a}$, Mark Widholm ${ }^{a}$, \\ Tomohiko Narita ${ }^{b}$, Louis-André Hamel ${ }^{c}$ \\ ${ }^{a}$ Space Science Center, University of New Hampshire, 39 College Road, Durham, NH USA \\ 03824; \\ ${ }^{b}$ Dept. of Physics, College of the Holy Cross, 1 College Street, Worcester, MA USA 01610; \\ ${ }^{c}$ Dept. of Physics, University of Montreal, P.O. Box 6128 Downtown Station, Montreal, \\ Quebec, Canada, H3C 3J7
}

\begin{abstract}
We report progress in the study of a thick CZT strip detector module designed to perform gamma-ray spectroscopy and 3-D imaging. We report preliminary performance measurements of $7.5 \mathrm{~mm}$ thick single-sided charge-sharing strip detector prototype devices. This design features both row and column contacts on the anode surface. This electron-only approach addresses problems associated with poor hole transport in CZT that limit the thickness and energy range of double-sided strip detectors. This work includes laboratory and simulation studies aimed at developing compact, efficient, detector modules for 0.05 to $1 \mathrm{MeV}$ gamma measurements while minimizing the number and complexity of the electronic readout channels. This is particularly important in space-based coded aperture and Compton telescope instruments that require large area, large volume detector arrays. Such arrays will be required for the NASA Black Hole Finder Probe (BHFP) and Advanced Compton Telescope (ACT). This new design requires an anode pattern with contacts whose dimensions and spacing are roughly the size of the ionization charge cloud. The first prototype devices have $125 \mu \mathrm{m}$ anode contacts on $225 \mu \mathrm{m}$ pitch. Our results demonstrate the principle of operation but suggest that even finer anode contact feature sizes will be necessary to achieve the desired performance.
\end{abstract}

Keywords: CZT, strip detectors, gamma-ray

\section{INTRODUCTION}

Cadmium Zinc Telluride (CZT) detectors have desirable features for high performance gamma-ray imaging spectrometers. The poor hole transport properties of CZT, however, must be considered when designing detectors thicker than a few millimeters. Effective designs for thicker detectors employ small contact features on the anode surface. $^{1-3}$ These single-sided or electron-only devices include pixel and strip detectors. The advantages of single sided strip detector designs are as follows.

1. An NxN pixel detector requires $\mathrm{N}^{2}$ channels whereas a single-sided CZT strip detector requires only $2 \mathrm{~N}$ channels. This feature is important in construction of large detector arrays because fewer channels draw less power and reduce the complexity of instrument electronics.

2. Since all signals are on one side of the detector, the design and fabrication of closely packed arrays is simpler than for double-sided strip detectors where the electrical contacts must be instrumented on both surfaces.

3. Because electron transport is efficient, single-sided strip detectors can be made up to $10 \mathrm{~mm}$ thick, so they can be used effectively in the range 0.05 to $1 \mathrm{MeV}$. On the other hand, double-sided strip detectors can be fabricated only up to $3 \mathrm{~mm}$ which limits their use up to $0.3 \mathrm{MeV}$.

A single sided strip detector design has also been applied to silicon. ${ }^{4}$

Further author information: (Send correspondence to B.D.)

B.D.: E-mail: bdonmez@comptel.sr.unh.edu, Telephone: 16038622756

Hard X-Ray and Gamma-Ray Detector Physics VII, edited by Ralph B. James, Larry A. Franks,

Arnold Burger, Proceedings of SPIE Vol. 5922 (SPIE, Bellingham, WA, 2005)

0277-786X/05/\$15 - doi: 10.1117/12.617297

Proc. of SPIE Vol. $592259220 \mathrm{H}-1$ 


\section{SINGLE-SIDED CHARGE-SHARING STRIP DETECTORS}

\subsection{Detector concept and first prototype}

Figure 1 shows a charge-sharing CZT strip detector ${ }^{1,2}$ with eight row channels and eight column channels (64 "pixels" or "unit cells"). The anode pattern and two $1.225 \mathrm{~mm}$ square unit cells (expanded right) illustrate the pad interconnections. Each unit cell contains an array of closely packed anode contact pads in two groups (gray and black in this figure). The two groups are identically biased for electron charge collection but are interconnected in columns and rows in the layers of the carrier substrate. A non-collecting grid electrode surrounding each pixel, biased between pixel pad and cathode, provides a signal that can be used for measuring the depth of interaction (the Z-coordinate). A single cathode contact on the opposite side is not shown. The principle of operation requires a sharing of charge between row and column anode contacts for each event. This is feasible when the pitch size of the anode pads are smaller than the lateral extent of the electron charge cloud reaching the anode surface. This approach takes advantage of the increasing capability of manufacturers to pattern and interconnect fine features.

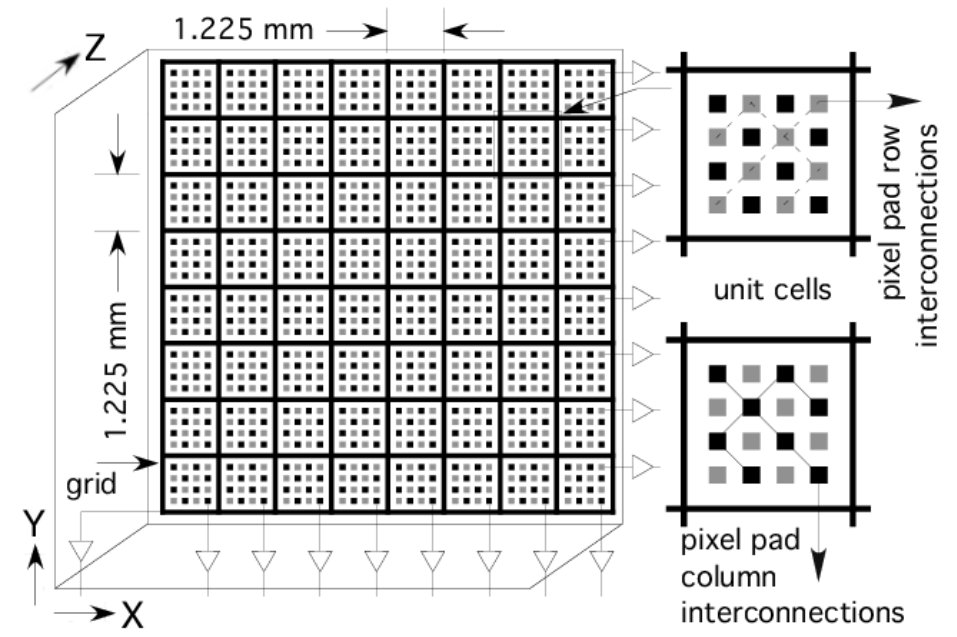

Figure 1. Single-sided charge-sharing strip detector (left). Unit cells (right) show interconnections.

An important advantage of this design is the simplicity of the front-end electronics. Polarities and shaping times (and ASICs) are the same for both row and column channels. Since row and column electrodes are identically biased, surface leakage between them vanishes. Also, the large area covered by the grid electrode should result in better depth resolution than was available from the individual strip column electrodes in our earlier single-sided strip detector design. ${ }^{2}$

There are some disadvantages with this design. To measure energy, column and row signals must be added. This degrades the achievable energy resolution by a factor related to the electronic noise. Capacitance effects due to the contact pad and interconnect structure also increase the noise. However, selecting the proper ASIC may minimize this effect. We also anticipate that limited charge sharing due to the small size of the electron cloud, particularly at low energies, will for some events, result in a measurement of only X or Y, not both, and will, at least for the first prototype detectors reduce the detection efficiency for imaging measurements.

Photographs of the components of an 11 row $\times 11$ column prototype detector module are shown in Figure 2. Pixel pitch is $1.225 \mathrm{~mm}$ for both rows and columns. The patterned CZT anode surface is shown in Figure 2(a). The top surface of the ceramic substrate is shown in Figure 2(b). Figure 2(c) shows the bottom surface of the ceramic substrate designed for us by MillPack, Inc. It was formed using Dupont Fodel layers on an alumina substrate. Multiple Fodel layers provide the mating surface contacts, the interconnection of the row contacts, the interconnection of the column contacts, a shield layer to reduce signal coupling between rows and columns, vias 


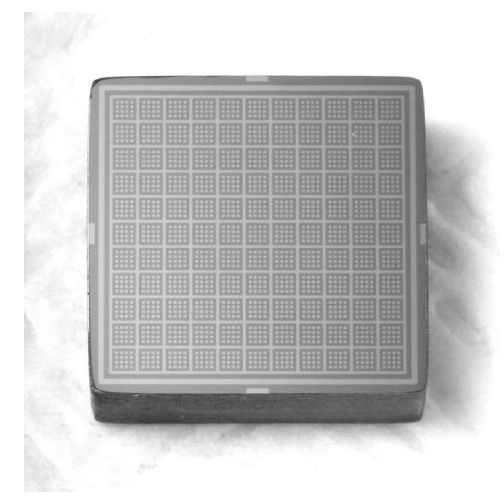

(a)

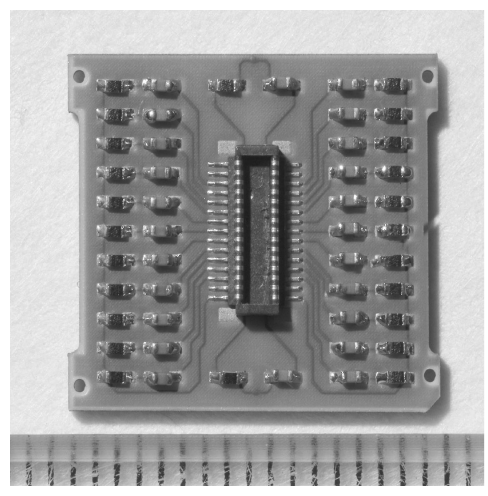

(c)

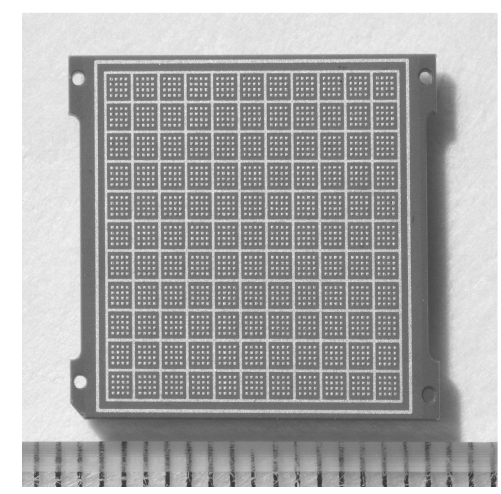

(b)

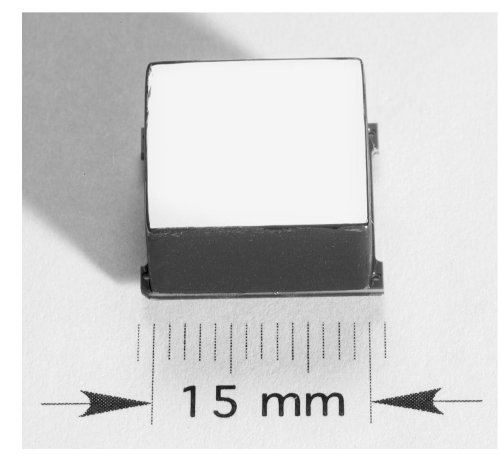

(d)

Figure 2. Components of a prototype detector module. CZT anode (a); anode mating surface of ceramic subsrate (b); bottom of ceramic substrate with passive components and connector (c). Detector module assembly (d). Ruler marks are $1 \mathrm{~mm}$.

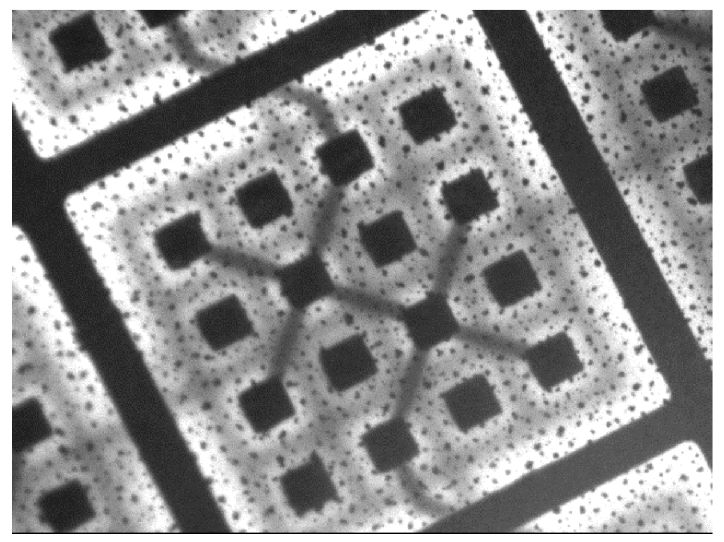

Figure 3. One unit cell pattern on the ceramic substrate viewed through an eV Products Z-bond sample.

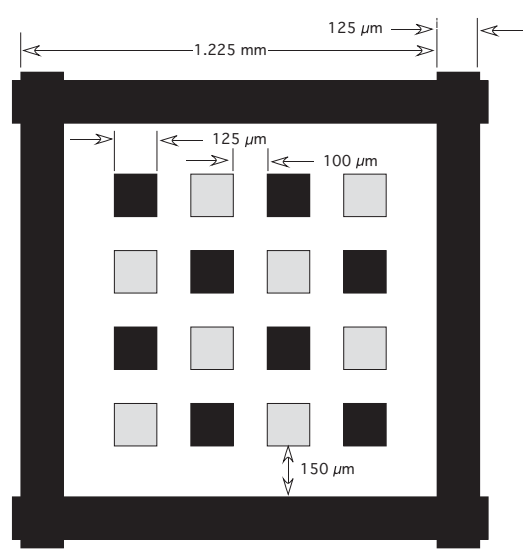

Figure 4. The actual contact sizes of a unit cell. 
to interconnect the layers and routing of row and column signal and biases to and from the passive components and the connector (integrated on the underside). Figure 2(d) shows an assembled detector module.

The eV Products Z-bond process was used to bond the $7.5 \mathrm{~mm}$ thick patterned CZT substrate to the ceramic substrate. A photograph through glass of a Z-bond sample layer on a unit cell of a ceramic substrate is shown in Figure 3. The black dots are the metal filaments that electrically connect the contact pads on the mating surfaces. The contact and gap sizes for a single unit cell are shown in Figure 4.

Electronic noise, spectroscopic performance and the efficiency for X-Y imaging have now been evaluated with a prototype device. The results are presented below.

\subsection{Spectroscopy}
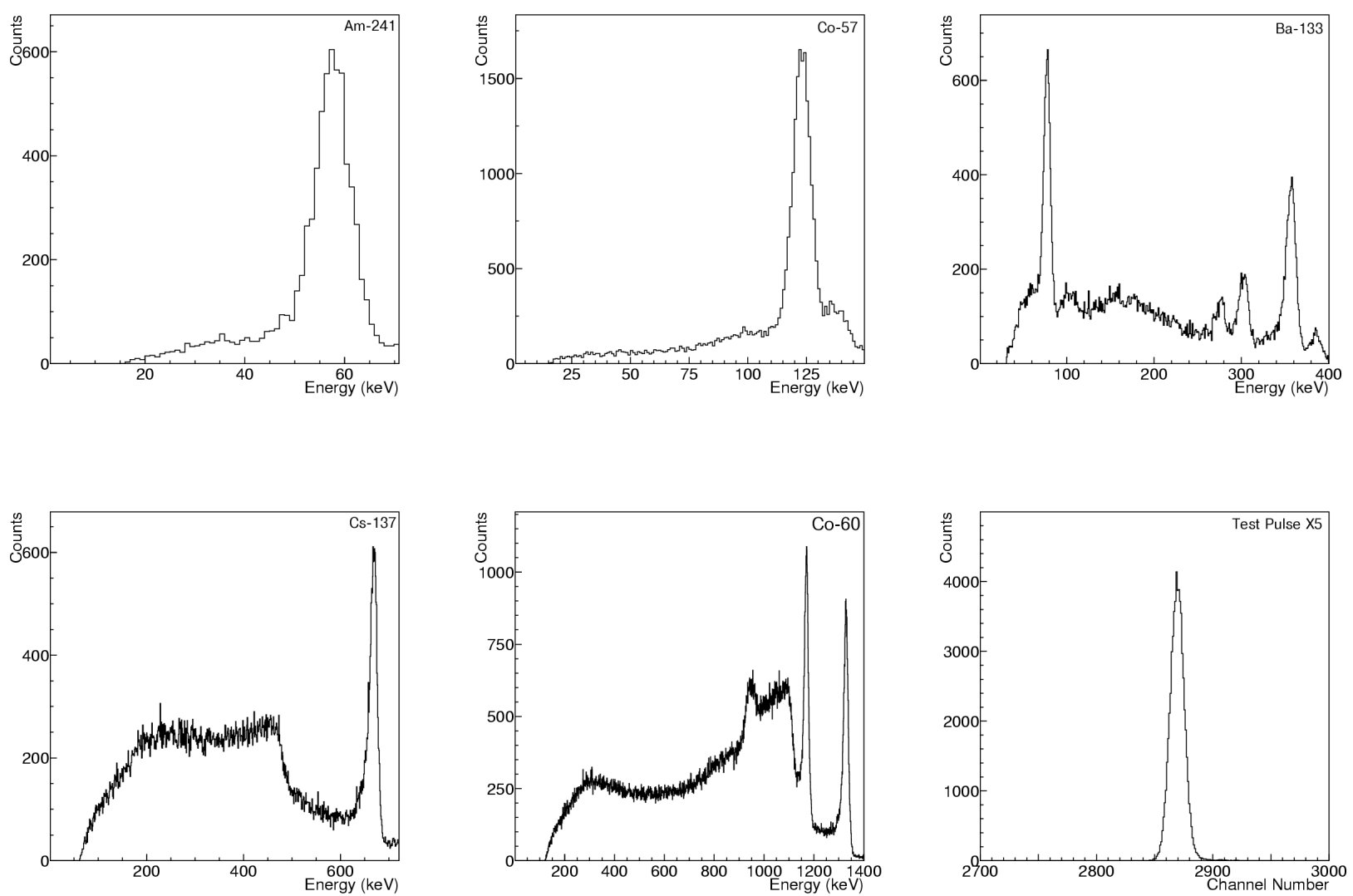

Figure 5. Spectroscopic performance and test pulse response of a unit cell of a single-sided charge-sharing strip detector. Spectra construction explained in the text.

Spectra from flood illumination of a prototype detector at room temperature with calibration source photons spanning the energy range from 60 to $1333 \mathrm{KeV}$ are shown in Figure 5. These single pixel spectra were constructed from the addition of the maximum row and maximum column pulse heights (here we selected row 5 column 5 , i.e. X5 and Y5). We have applied a threshold value for both $\mathrm{X}$ and $\mathrm{Y}$ pulse heights in the software to obtain these spectra. Threshold values are $8,8,16,32$ and $64 \mathrm{keV}$ at $60,122,356,662,1333 \mathrm{keV}$ respectively. We have not used any pulse height correction for interaction depth. Energy resolution (FWHM) is 9.4, 10.1, 13.6, $19.5,23.0$ and $23.7 \mathrm{keV}$ at $60,122,356,662,1173$ and $1333 \mathrm{keV}$ respectively. The photopeaks are symmetric with no significant low energy tailing that would indicate a loss of signal to the non-collecting areas of the anode surface. The electronic noise is $5.7 \mathrm{keV}$ FWHM per channel, or $8.0 \mathrm{keV}$ (FWHM) for the combined row and 
column signals. We note that the summed spectra have broader peaks than measured with an earlier design. ${ }^{2}$ This is partly due to the addition in quadrature of the noise component of the row and column signals.

\subsection{Imaging}

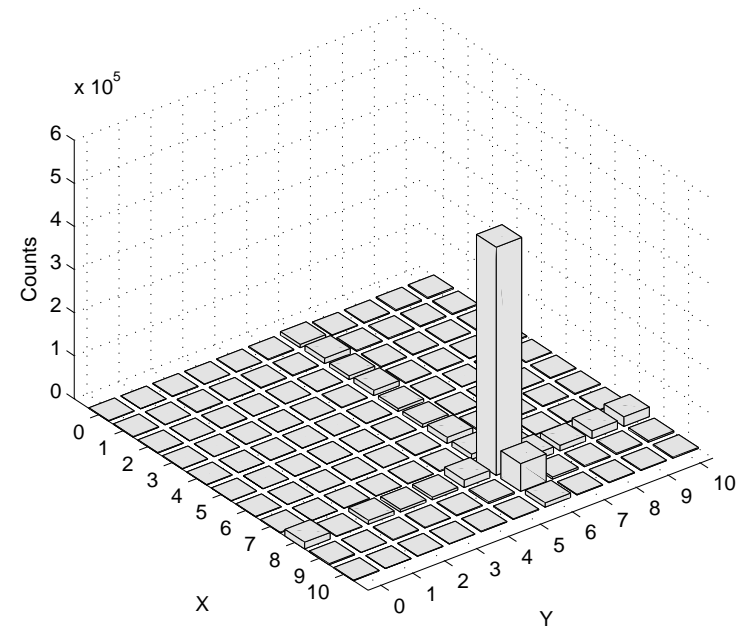

(a)

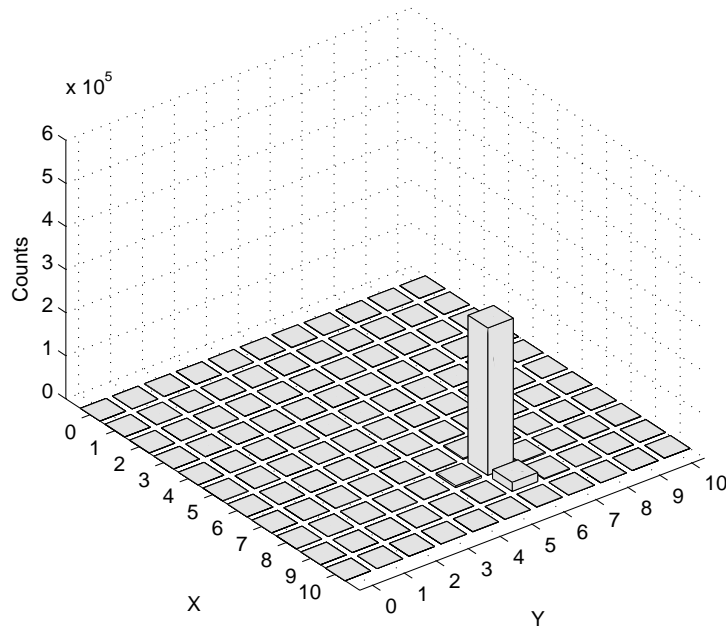

(b)

Figure 6. Computed event locations for collimated ${ }^{57}$ Co beam spot. For all events (a); for events above sharing threshold (b).

Charge sharing between rows and columns plays an important role in determining the imaging efficiency for this design. Both row and column signals must exceed the noise threshold to achieve a measure of the $\mathrm{X}$ and Y location. To measure the fraction of events registering sufficient signal on either row or column channels, we made a run with a collimated ${ }^{57} \mathrm{Co}$ source. Figure 6 shows the computed event location for a $1 \mathrm{~mm}$ diameter beam of ${ }^{57}$ Co photons. The computed image of all photopeak events $(122 \mathrm{keV})$ is shown on the left (Figure $6(\mathrm{a})$ ). The image on the right (Figure 6(b)) is formed from events whose row and column pulse heights exceeded the $8 \mathrm{keV}$ threshold. We find that $64 \%$ of events are above this threshold for both row and column. This fraction represents the efficiency for imaging measurement at this energy. Studies are underway to help us understand the sources of inefficiency and to develop improved designs. Figure 7 shows scatter plots for each calibration source. We see that at all energies, there is a significant number of events for which most or all of the charge is collected on one channel only (row or column). In an ideal charge-sharing detector, however, events on the scatter plot should accumulate along the main diagonal with comparable signals in both $\mathrm{X}$ and $\mathrm{Y}$.

\section{SIMULATIONS}

To help understand why our prototype device falls short of this expectation, we performed GEANT (v4.6) simulations to determine the initial charge cloud radius and calculated the expected effects of diffusion and electrostatic repulsion of the charge as it drifts toward the anode surface.

\subsection{Size of the Charge Cloud}

The radius of the charge cloud reaching the anode for any interaction depends on the type of interaction (photoelectric or Compton), the energy deposit and the depth of interaction. ${ }^{5}$ In order to register sufficient signal on both row and column channels, the extent of the electron charge distribution reaching the anode surface must be at least as large as the contact pad pitch, $225 \mu \mathrm{m}$ for our prototype devices. Our simulation results, however, 

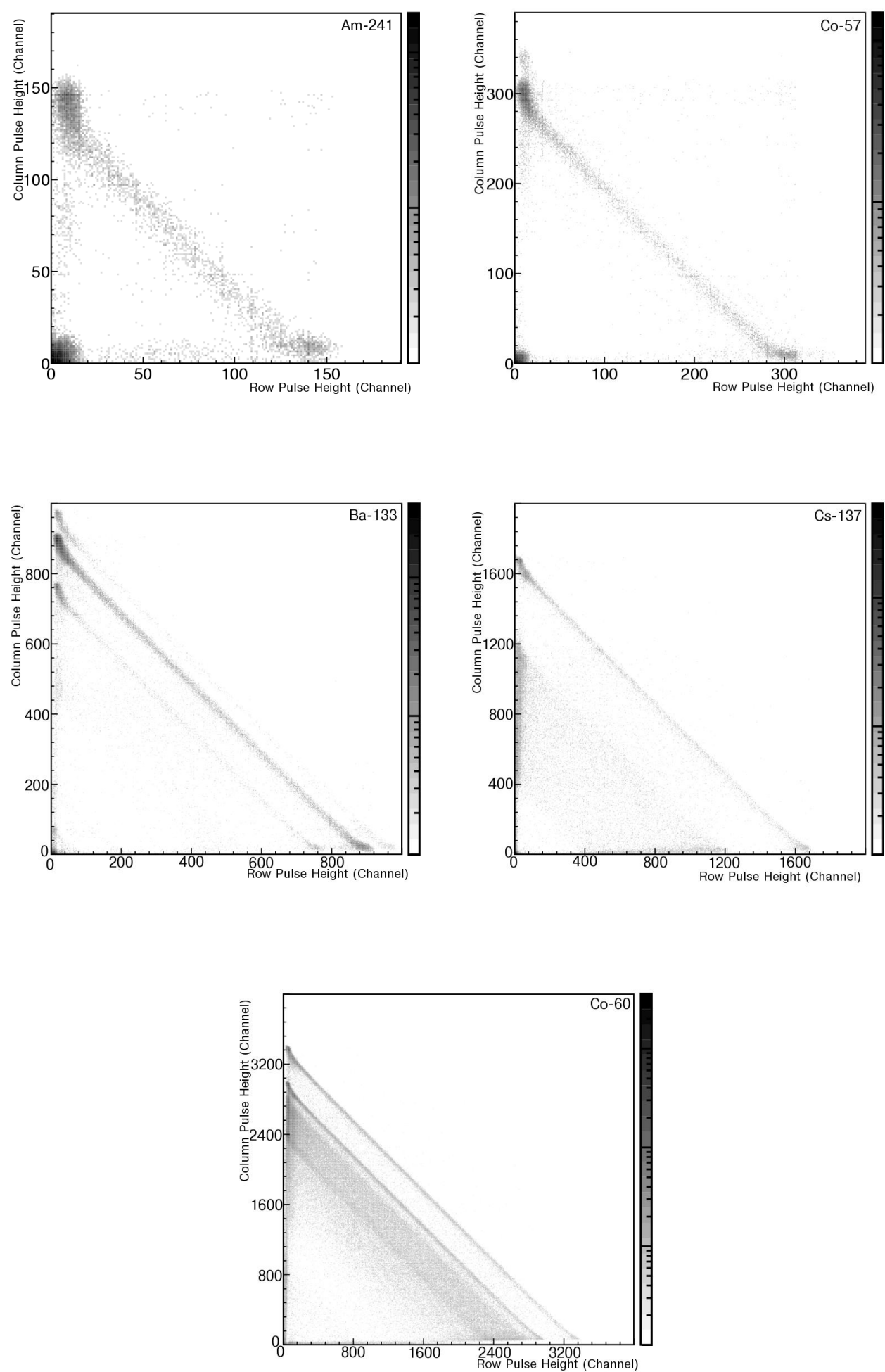

Figure 7. Scatter plots for X5 and Y5 signals for each calibration source.

Proc. of SPIE Vol. $592259220 \mathrm{H}-6$ 
show that, on average, $95 \%$ of the energy is concentrated within only a $100 \mu$ m radius at $100 \mathrm{keV}$. At $1500 \mathrm{keV}$, the average radius for $95 \%$ energy enclosure is $\sim 400 \mu \mathrm{m}$.

Figure 8, shows a GEANT simulated scatter plot of row versus column charge sharing for $122 \mathrm{keV}$ photons $\left({ }^{57} \mathrm{Co}\right)$. This simulation is analogous to the prototype detector's anode pad and gap dimensions (Figure 4$)$ and the measurements shown in Figure 7. The simulation is setup so that photons randomly hit a $225 \times 225 \mu \mathrm{m}^{2}$ area $(125 \mu \mathrm{m}$ pad $+2 \times 50 \mu \mathrm{m}$ half-gap) centered on a pad. For each event the simulation computes a map of the deposited energy (ionization charge) and its geometric projection on the anode plane. The energy projected is divided between row and column ( $\mathrm{X}$ and $\mathrm{Y}$ ) regions of the anode surface. If more than $8 \mathrm{keV}$ is collected by one of the orthogonal direction pads, that event is considered "X-Y shared". No instrument noise was included. The simulated result shows that only $26 \%$ of the events are tagged as shared events. These are the events distributed along the sloping line. Measurements, however, at this energy with our prototype detectors (Figure 6) yield a much higher fraction of "shared" events (64\%). This indicates that effects other than the extent of the ionization at the interaction site are significant factors. Lateral diffusion and electrostatic repulsion of the electrons as they migrate to the anode surface is one effect that are two effects that merit further consideration.

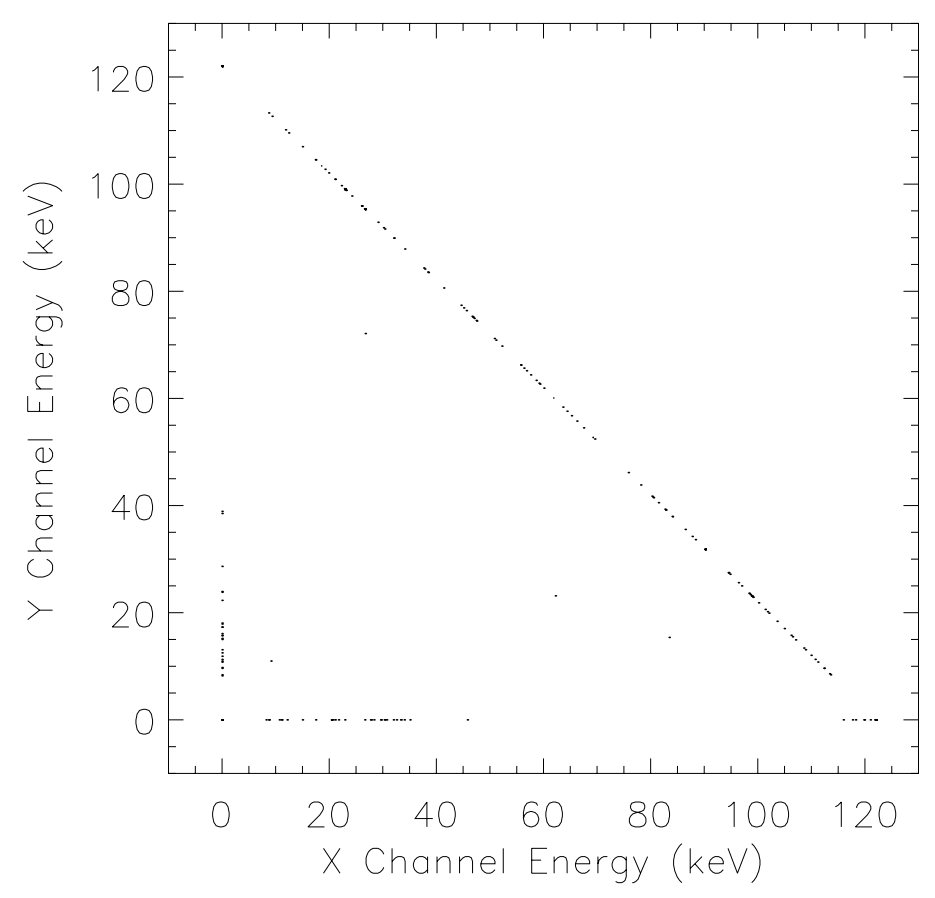

Figure 8. GEANT simulated scatter plot of X and $\mathrm{Y}$ signals for $122 \mathrm{keV}$ photons $\left({ }^{57}\right.$ Co.).

\subsection{Diffusion and Electrostatic Repulsion}

Diffusion of the charge cloud as it moves toward the anode surface will increase the extent of the charge distribution. The diffusion coefficient is related to the mobility and the temperature through Einstein's relation so that, at room temperature, the diffusion coefficient for electrons in CZT is approximately $25 \mathrm{~cm}^{2} / \mathrm{s}$. The width of the distribution due to the diffusion must be added in quadrature with the width of the initial charge distribution to obtain the spread of the charge distribution. It is also seen that even for low ionization densities, electrostatic repulsion between the electrons plays a significant role for silicon detectors in the size of the cloud when it reaches the anode plane. ${ }^{6}$ As the cloud expands as the square root of time under the effect of diffusion, it also expands 
as the cubic root of time with repulsion. It appears that repulsion may become the dominant effect at short times and that diffusion becomes more important when the cloud density decreases. The diffusion and repulsion processes are decoupled and their contributions to the cloud size add quadratically.

In addition to these effects, electrons will drift in the direction of the electric field. In a uniform electric field, all these effects are decoupled and the movement of the electrons is given by an expanding cloud that otherwise drift at a uniform speed.

Several groups have studied the charge spreading in semiconductor detectors. A study of the position resolution of stripixel detectors show that the pitch must be no greater than the size of the charge carrier cloud and that charge spreading improves the position resolution. ${ }^{7}$ The spread has also been measured for germanium detectors. ${ }^{8}$ There are also studies on $\mathrm{CdTe}^{9,10}$ and $\mathrm{CdZnTe}^{5}$ detectors.

Considering these as well as our preliminary studies, we currently estimate, at $100 \mathrm{keV}, 25 \mu \mathrm{m}$ rms for the initial size of the electron cloud, $25 \mu \mathrm{m}$ for diffusion and $50 \mu \mathrm{m}$ for electrostatic repulsion. This yields $60 \mu \mathrm{m} \mathrm{rms}$ or $140 \mu \mathrm{m}$ FWHM when all effects are added quadratically. This figure represents a reasonable target for the contact pad pitch in subsequent detector designs.

\section{CONCLUSIONS AND FUTURE WORK}

Our goal is to develop and demonstrate compact, efficient, high performance CZT strip detector modules for imaging and spectroscopy for 0.05 to $1 \mathrm{MeV}$ gamma measurements and be ready to employ them in large area detector arrays when large volumes of suitable CZT material with uniform properties become available and affordable.

We have designed a new type CZT detector, the single-sided charge sharing strip detector, and fabricated the first prototypes for evaluation in the laboratory. These prototype devices feature $125 \mu \mathrm{m}$ anode contact pads on $225 \mu \mathrm{m}$ pitch and require signal charge to be shared among row and column pads in order to perform imaging measurements. The test results demonstrate good spectroscopic response even though the row and column signals must be summed to construct the spectra. We have demonstrated 2-D imaging using row and column contacts on a single detector surface and measured X-Y imaging efficiency to be greater than $64 \%$ above $60 \mathrm{keV}$.

We have developed Monte-Carlo simulation tools based on GEANT (v4.6) in part to help us understand the extent of the ionization and as an aid to guiding future detector design. The simulations indicate that the lateral extent of the charge at the ionization site is significantly smaller than the contact pad pitch of our first prototype devices. Tests with these prototypes, however, demonstrate greater imaging efficiency than can be accounted for by these simulation results alone. We suspect that lateral charge diffusion and electrostatic repulsion play significant roles in imaging efficiency and have estimated their contributions. We expect that significantly greater $\mathrm{X}$-Y imaging efficiency will be achieved with a modified detector design featuring contact pads on $150 \mu \mathrm{m}$ or finer pitch.

We will continue to use simulation tools to help us understanding the performance of our detectors. Future work includes extending simulation to include diffusion and repulsion effects and to extend the laboratory study to 3-D imaging. We will also develop and test prototype detectors with smaller anode contact features. We will pursue an improved design with smaller anode contact features.

\section{ACKNOWLEDGMENTS}

This work was supported in the USA by NASA's High Energy Astrophysics Supporting Research and Technology Program under Grant No NNG05WC25G, and in Canada by the Natural Sciences and Engineering Research Council. 


\section{REFERENCES}

1. J. Macri, L.-A. Hamel, M. Julien, B. Dönmez, M. L. McConnell, J. M. Ryan, M. Widholm, and T. Narita, "Single-sided Charge-Sharing CZT Strip Detectors," presented at IEEE Nuclear Science Symposium and Medical Imaging Conference, Rome, Italy, 2004.

2. J. Macri, B. Dönmez, M. Widholm, L.-A. Hamel, M. Julien, T. Narita, J. Ryan, and M. McConnell, "Singlesided CZT Strip Detectors," SPIE, 5501, p. 208, 2004.

3. Z. He, W. Li, G.F. Knoll, D.K. Wehe, J. Berry, and C.M. Stahle, "3-D position sensitive CdZnTe gamma-ray spectrometers", NIM A, 422, p. 173, 1999.

4. Z. Li, "Novel silicon stripixel detector: concept, simulation, design, and fabrication", NIM A, 518, p. 738, 2004.

5. E. Kalemci and J. L. Matteson, "Investigation of Charge Sharing Among Electrode Strips for a CdZnTe Detector," NIM A, 478, p. 529, 2002.

6. E. Gatti, A. Longoni, P. Rehak, and M. Sampietro, "Dynamics of Electrons in Drift Detectors", NIM A,253, p. 393, 1987.

7. B. Yu et al., "A Novel 2D Position Sensitive Silicon Detector with Micron Resolution for Heavy Ion Tracking", presented at IEEE Nuclear Science Symposium and Medical Imaging Conference, Rome, Italy, 2004.

8. R. A. Kroeger, "Charge Spreading and Position Sensitivity in a Segmented Planar Germanium Detector", NIM A,422, p. 206, 1998.

9. R. Miyawaki et al., "High Resolution Fourier Synthesis Hard X-Ray Imaging Based on CdTe Strip Detectors", presented at IEEE Nuclear Science Symposium and Medical Imaging Conference, Rome, Italy, 2004.

10. G. Pellegrini et al., "Performance Limits of a $55 \mu \mathrm{m}$ Pixel CdTe Detector", presented at IEEE Nuclear Science Symposium and Medical Imaging Conference, Rome, Italy, 2004.

Proc. of SPIE Vol. $592259220 \mathrm{H}-9$ 